\title{
Liste des figures
}

Figure 1.1 : Schéma général de la formation du physicien médical en France en 2011

Figure 1.2 : Articulation générale des tutelles administratives, directes et indirectes, relatives à l'utilisation médicale des rayonnements ionisants

Figure 1.3 : Schéma général des relations entre les différentes exigences servant de base à la réglementation

Figure 2.1 : Articulation entre les différents systèmes de gestion de la qualité

Figure 2.2 : Les 5 axes des cyndiniques (d'après Kervern 1995)

Figure 2.3 : Principe général d'utilisation des données

Figure 2.4 : Schéma d'organisation du retour d'expérience (REX)

Figure 2.5 : Illustration de la défense en profondeur vis-à-vis du risque

d'erreur d'étalonnage pour un appareil de télécobaltothérapie

Figure 3.1 : Différentes représentations graphiques des variations

de la valeur de l'unité moniteur (cGy/MU) sur une période donnée

Figure 3.2 : Exemple de carte de contrôle de la valeur des unités moniteurs

Figure A.1 : Schéma général proposé pour homogénéiser la qualification

des physiciens médicaux en Europe 
Guide des bonnes pratiques de physique médicale

Figure A.2 : Hiérarchie des tableaux décrivant les résultats d'apprentissage requis pour pouvoir obtenir la reconnaissance en tant que "Medical Physics Expert » (MPE)

Figure A.3 : Schéma de la roue de Deming 